\title{
Enhanced hepatic differentiation of rat bone marrow-derived mesenchymal stem cells in spheroidal aggregate culture on a decellularized liver scaffold
}

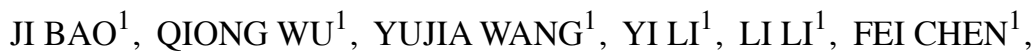 \\ XIUJUAN WU ${ }^{3}$, MINGJUN XIE ${ }^{3}$ and $\mathrm{HONG} \mathrm{BU}^{1,2}$ \\ ${ }^{1}$ Laboratory of Pathology, ${ }^{2}$ Department of Pathology, West China Hospital, Sichuan University, Chengdu, Sichuan 610041; \\ ${ }^{3}$ Department of General Surgery, Yibin City First People's Hospital, Yibin, Sichuan 644000, P.R. China
}

Received May 8, 2015; Accepted June 1, 2016

DOI: $10.3892 / \mathrm{ijmm} .2016 .2638$

\begin{abstract}
In the present study, we aimed to determine whether the combination of aggregate culture and decellularized liver scaffolds (DLSs) promoted the hepatic differentiation of murine bone marrow-derived mesenchymal stem cells (BM-MSCs) into high yields of mature hepatocytes in vitro. Four culturing methods for differentiation [single cell (2D), spheroids (3D), 2D + DLS and $3 \mathrm{D}+\mathrm{DLS}$ ] were studied. To determine the differentiation stages of the MSCs, RT-qPCR of the hepatocyte genes, immunostaining of hepatocyte markers, and functional analyses were all performed. Compared with the other groups, hepatocyte-like cells which differentiated from BM-MSC spheroids on extracellular matrix (ECM) exhibited more intensive staining of stored glycogen, an elevated level of urea biosynthesis and albumin secretion as well as the higher expression of hepatocyte-specific genes. Our results indicated that DLSs combined with spheroidal aggregate culture may be used as an effective method to facili-
\end{abstract}

Correspondence to: Dr Hong Bu, Laboratory of Pathology, West China Hospital, Sichuan University, 37 Guoxue Road, Chengdu, Sichuan 610041, P.R. China

E-mail: hongbu@scu.edu.cn

Abbreviations: MSCs, mesenchymal stem cells; BM-MSCs, bone marrow-derived MSCs; 2-D, two-dimensional; 3-D, three-dimensional; ECM, extracellular matrix; DLS, decellularized liver scaffold; H\&E, hematoxylin and eosin; DAPI, 4',6-diamidino-2-phenylindole; GAGs, glycosaminoglycans; SEM, scanning electron microscopy; IMDM, Iscove's modified Dulbecco's medium; EGF, epidermal growth factor; bFGF, basic fibroblast growth factor; HGF, hepatocyte growth factor; EdU, 5-ethynyl-2'-deoxyuridine; RT-qPCR, reverse transcription quantitative-polymerase chain reaction; OCT, optimum cutting temperature; PBS, phosphate-buffered solution; AFP, alpha fetoprotein; ALB, albumin; CK19, cytokeratin 19; PAS, periodic acid-Schiff; SDS, sodium dodecyl sulfate; HNF1 $\beta$, hepatocyte nuclear factor $1 \beta$; HNF6, hepatocyte nuclear factor 6; TAT, tyrosine aminotransferase; FOXA1, Forkhead box A1; ARG1, arginase 1

Key words: mesenchymal stem cells, decellularization, spheroid, liver extracellular matrix, hepatic differentiation tate the hepatic maturation of BM-MSCs and may have future applications in stem cell-based liver regenerative medicine.

\section{Introduction}

Orthotopic liver transplantation is the only currently available treatment for severe liver failure; however, the use of this technique is limited by organ shortages (1). Hepatocyte transplantation has become an attractive alternative approach to treating liver diseases (2). However, the isolation of sufficient numbers of transplantable hepatocytes is restricted by the small number of marginal donor organs (3). In addition, adult human hepatocytes have poor proliferative potential that is likely to be insufficient for effectively repopulating the host liver (4). The search for greater cell resources, which may be used to replace primary hepatocytes, has prompted investigations into the generation of hepatocytes from stem cells of extrahepatic origin, owing to their ready availability and unrestricted ability to propagate and differentiate (5). Mesenchymal stem cells (MSCs), which are found in various tissues and organs, have become an important resource for regenerative medicine, as they are readily available cells that proliferate in vitro and have the capacity for multiple lineage differentiation (6). Previous studies using rats, mice and humans have confirmed that bone marrow-derived MSCs (BM-MSCs) as well as MSCs derived from umbilical cord blood or adipose tissue may differentiate into hepatocyte-like cells under selective growth conditions in vitro, which suggests that adult stem cellbased therapies may provide alternative therapeutic approaches for the treatment of liver diseases (7-9).

Considerable efforts over the last decade have been dedicated to the evaluation of culture conditions in order to obtain differentiated hepatocytes from stem cells. The sequential addition of liver-specific factors into culture systems in a time-dependent manner that mimics the secretion pattern during liver embryogenesis is important for the differentiation of stem cells into hepatocytes (10). Advances in induction technology have enabled the in vitro differentiation of MSCs into hepatocyte-like cells using a two-dimensional (2D) culture system (11). However, the 2D culture method has limitations in terms of controlling stem cell differentiation pathways, resulting in low differentiation efficiency. Three-dimensional (3D) culture 
systems have been shown to promote enhanced cellular structure and function in many types of cells and tissues, including mammary epithelial cells, MSCs as well as neural and hepatic cells (12-14). MSCs cultured as 3D spheroids in suspension are characterized by enhanced levels of differentiation and higher degrees of maturity of MSC-derived hepatocytes compared with traditional adherent monolayer cultures (15-17).

Bio-scaffolds derived from decellularized organ/tissue matrix have been used for the differentiation of stem cells due to the preserved extracellular matrix (ECM) components, which include numerous chemical and biophysical cues for differentiation $(18,19)$. In addition, previous findings have shown that the differentiation of stem/progenitor cells is lineage restricted by the tissue-specific biomatrix scaffold $(18,19)$. Therefore, a decellularized liver may potentially be used as a tool for stem cell differentiation and maturation, and eventually be used to engineer autologous liver grafts. Previous studies have demonstrated that the differentiation of stem cells derived from different tissues into hepatocyte-like cells is more efficient in a decellularized liver biomatrix $(20,21)$.

As interactions between stem cells and the ECM are required for inducing lineage-specific differentiation and maintaining the biological functions of hepatocyte-like cells by providing a composite set of chemical and structural signals, in the present study we employed both 3D spheroid and decellularized liver scaffold (DLS) culture systems to promote hepatocyte maturation of the hepatocyte-like cells. This combination is a novel method whereby rat BM-MSCs self-aggregated into spheroids in $3 \mathrm{D}$ culture plates and were then implanted into the DLS.

\section{Materials and methods}

Animals.Male Bama miniature pigs (Guangxi, China) weighing 10-12.5 kg were obtained from the Animal Experiment Center of Sichuan University (Chengdu, China), and the whole liver was harvested. The animals were maintained under a 12-h light/dark cycle with free access to standard laboratory food and water. All experimental protocols were approved by the Animal Experiment Center of Sichuan University. All animals were cared for in accordance with the requirements of the Laboratory Animal Welfare Act and amendments.

Six livers were isolated from male Bama miniature pigs for perfusion decellularization. The surgeries were performed under ketamine ( $6 \mathrm{mg} / \mathrm{kg}$ body weight, administered IP; Kelun, Chengdu, China) and xylazine (10 mg/kg IP; Kelun) anesthesia. Under deep anesthesia, a laparotomy was performed and the liver was exposed. After systemic heparinization through the inferior vena cava, the hepatogastric ligament was carefully dissected. The proximal PV was catheterized. The hepatic artery and common bile duct were ligated and transected. All perihepatic ligaments were severed. Simultaneously, the liver was slowly perfused with 2 liters of deionized water containing $0.1 \%$ EDTA (Kelun) through a cannula in the PV, and the SHIVC was transected, allowing outflow of the perfusate. Following blanching, the liver was stored at $-80^{\circ} \mathrm{C}$ overnight.

Evaluation of decellularized porcine liver. We used our previously established decellularization protocol to obtain liver scaffolds (22). The liver was perfused with $1 \%$ Triton X-100 (Amresco, Solon, OH, USA) for $3 \mathrm{~h}$ and then by $1 \%$ SDS
(Promega, San Luis Obispo, CA, USA) in deionized water at a rate of $200 \mathrm{ml} / \mathrm{min}$ for $6 \mathrm{~h}$ after thawing. This was followed by $3 \mathrm{~h}$ of perfusion with $1 \%$ Triton X-100 to remove residual SDS. Subsequently, the liver was washed with 20 liters of distilled water to remove residual detergent, followed by infusion of 40 liters of phosphate-buffered saline (PBS) at $200 \mathrm{ml} / \mathrm{min}$. To determine whether collagen I $(1: 1,000$, mouse polyclonal IgG, GTX26308; GeneTex, Irvine, CA, USA); collagen IV (1:100, rabbit polyclonal IgG, bs-4595R; BIOSS, Beijing, China); laminin (1:1,000, mouse polyclonal IgG, GTX11574) and fibronectin (1:100, rabbit polyclonal IgG, GTX72724) (both from GeneTex) were retained in the decellularized matrices, the liver ECM samples were sectioned and stained by immunohistochemistry with the indicated antibodies and dilutions. Briefly, paraffin sections were rehydrated, incubated in antigen retrieval solution, and stained using antibodies to fibronectin, laminin, and collagen I and IV. Images of the stained slides were captured using an upright microscope (BX51; Olympus, Tokyo, Japan). Sulfated glycosaminoglycans (GAGs) were quantified using the Blyscan GAG assay kit (Biocolor, Carrickfergus, UK).

Histological analysis. Normal fresh liver ( $\mathrm{n}=6$ of each group), decellularized liver matrix, and recellularized liver samples were fixed in $4 \%$ paraformaldehyde at room temperature for $24 \mathrm{~h}$. They were dehydrated using a graded ethanol series, immersed in xylene, and embedded in paraffin. The ECM samples were cut into $5-\mu \mathrm{m}$ sections and stained with hematoxylin and eosin $(\mathrm{H} \& \mathrm{E})$. Sections were mounted in mounting media containing 4',6-diamidino-2-phenylindole (DAPI; Santa Cruz Biotechnology, Inc., Dallas, TX, USA) to confirm the extent of decellularization.

Scanning electron microscopy (SEM). Following decellularization, the samples were fixed in $2.5 \%$ glutaraldehyde for at least $12 \mathrm{~h}$ at room temperature. The samples were then briefly rinsed in deionized water, dehydrated via a graded ethanol series, and dried in a critical point dryer (HCP2; Hitachi, Tokyo, Japan). Finally, the samples were sputter-coated with gold prior to SEM imaging. Electron micrographs of liver cross-sections were obtained at $5.0 \mathrm{kV}$ and x1,000 magnification using a Hitachi S-4800 scanning electron microscope (Hitachi).

Cultivation of rat BM-MSCs. Commercial rat BM-MSCs (Cyagen Biosciences, Guangzhou, China) at passages 6-8 (P6-8) were used in the following experiments. Sprague-Dawley rat MSC growth medium (no. RASMX-90011; Cyagen Biosciences) was used for cell culture. The medium was replaced at least every 2-3 days.

Hepatic differentiation of BM-MSCs in vitro. To induce hepatic differentiation, serum-free Iscove's modified Dulbecco's medium (IMDM; HyClone, Beijing, China) supplemented with a combination of growth factors described previously was used to induce the differentiation of BM-MSCs (7). Prior to the two-step induction protocol, the cells were serum-deprived for two days in IMDM supplemented with $20 \mathrm{ng} / \mathrm{ml}$ epidermal growth factor (EGF) and $10 \mathrm{ng} / \mathrm{ml}$ basic fibroblast growth factor (bFGF). The induction protocol was as follows: i) the BM-MSCs were treated with differentiation medium consisting of IMDM supplemented with $20 \mathrm{ng} / \mathrm{ml}$ hepatocyte growth 
Table I. RT-qPCR primer sequences.

\begin{tabular}{llll}
\hline Gene & $\begin{array}{c}\text { GenBank } \\
\text { Accesion No. }\end{array}$ & \multicolumn{1}{c}{ Forward primers $\left(5^{\prime} \rightarrow 3^{\prime}\right)$} & Reverse primers $\left(5^{\prime} \rightarrow 3^{\prime}\right)$ \\
\hline$H N F 1 \beta$ & NM_013103 & AATCCCAGCAAGGAAGAGAG & ACCAGTTGTAGACACGGACC \\
$H N F 6$ & NM_022671 & CCTGGAGCAAACTCAAGTCC & CCGTGTTCTTGCTCTTTCC \\
$T A T$ & NM_012668 & GGCACCTTCAGAAGATTTTG & GCCAGTGGTTCGTATTTGC \\
$F O X A 1$ & NM_012742 & GGTTCGGAGTTGAAGTCTCC & GGGGTGGTTAAAGGAGTAGTG \\
$C K 19$ & NM_199498 & GCCTACCTGAAGAAGAACCAC & CAATGCCTGGTGTGGAATC \\
AFP & NM_012493 & GCTGACAACATGGAGGAATG & TGAGTACAGCCTGGAGGTTC \\
ALB & NM_134326 & GGCACCAAGTGTTGTACCCT & AGCACACACAGACGGTTCAG \\
ARG1 & NM_017134 & CAACACTCCGCTGACAACC & CAGATATGCAGGGGGTCAC \\
$C Y P 1 A 1$ & NM_012540 & AGCTAATCAAAGAGCACTACAGG & CCTTATCATCTGAGAGCTGG \\
$C Y P 1 A 2$ & NM_012541 & GAGAAGGTGATGCTCTTCGG & ATGCAGGAGGATGGCTAAGA
\end{tabular}

$H N F 1 \beta$, hepatocyte nuclear factor $1 \beta ; H N F 6$, hepatocyte nuclear factor 6; TAT, tyrosine aminotransferase; FOXA1, forkhead box A1; CK19, cytokeratin 19; $A F P$, alpha fetoprotein; $A L B$, albumin; $A R G 1$, arginase 1; CYP1A1, cytochrome P450 family 1 subfamily A member 1 ; CYP1A2, cytochrome P450 family 1 subfamily A member 2.

factor (HGF), $10 \mathrm{ng} / \mathrm{ml} \mathrm{bFGF}$, and $0.61 \mathrm{~g} / 1$ nicotinamide (all from Sigma-Aldrich, St. Louis, MO, USA) for 7 days; ii) all groups were induced with maturation medium, which consisted of IMDM supplemented with $20 \mathrm{ng} / \mathrm{ml}$ oncostatin $\mathrm{M}, 1 \mathrm{mmol} / \mathrm{l}$ dexamethasone, and $50 \mathrm{mg} / \mathrm{ml}$ insulin-transferrin-selenium premix (all from Sigma-Aldrich) for 2 weeks.

Formation and characterization of $3 D$ BM-MSC spheroids Formation of BM-MSC spheroids. For spheroid cultures, the harvested BM-MSCs were suspended in $10 \mathrm{ml}$ serumfree medium at $1 \times 10^{6}$ cells $/ \mathrm{ml}$ and inoculated into glass spheroid dishes $(13 \times 8 \times 4 \mathrm{~cm})$ and were surface siliconized with Sigmacote (Sigma-Aldrich). The spheroid dishes were incubated with continuous rocking at $10 \mathrm{rpm}$ using the Rocker system (introduced by Mayo Clinic, Rochester, MN, USA) to induce spheroid formation, as previously described (23). After aggregation, $100 \mu \mathrm{l}$ aliquots were removed from the spheroid dishes to determine the number, diameter, and total volume (cell mass) of the spheroids using a Multisizer $3(560-\mu \mathrm{m}$ aperture; Beckman Coulter, Fullerton, CA, USA).

Cell viability assay. The viability of BM-MSC spheroids was evaluated using the FluoroQuench fluorescent viability stain (One Lambda, Canoga Park, CA, USA). The samples were imaged using a DFC 495 fluorescence microscope (Leica, Wetzlar, Germany).

5-Ethynyl-2'-deoxyuridine (EdU) staining. For EdU staining (cat. no. C10130-1; RiboBio, Guangzhou, China), BM-MSC spheroids were added to the respective culture flasks and cultured with $0.1 \%$ EdU overnight. EdU was probed using Apollo staining (RiboBio) thereafter.

Cell seeding. Four culturing methods for differentiation [single cell (2D), spheroids (3D), 2D + DLS and 3D + DLS] were studied. After the decellularization procedure, the decellularized livers were cut into discs of $8 \times 8 \times 3-\mathrm{mm}^{3}$ and placed into 24 -well plates for lyophilization, followed by sterilization with gamma irradiation (1,000 rad) for $2 \mathrm{~h}$. Prior to cell seeding, the discs were incubated in culture medium at $37^{\circ} \mathrm{C}$ overnight. After the medium was aspirated, a cell suspension $(100 \mu \mathrm{l})$ of harvested BM-MSCs or BM-MSC spheroids was pipetted onto the center of the disc. The cells were allowed to settle and attach to the disc scaffold for $4 \mathrm{~h}$. Subsequently, $2 \mathrm{ml}$ medium from stage one of the induction protocol was added slowly. Monolayer cells were cultured in normal 6-well plates as the control.

Reverse transcription-quantitative polymerase chain reaction (RT-qPCR). To harvest the differentiated cells or spheroids cultured in the DLS, the bio-scaffolds were washed twice with phosphate-buffered solution (PBS), chopped (cut into sections by opthalmic scissors) and digested with $1 \mathrm{mg} / \mathrm{ml}$ collagenase type II (Gibco, Grand Island, NY, USA) for $25 \mathrm{~min}$ at $37^{\circ} \mathrm{C}$. After filtration through a $200-\mu \mathrm{m}$ mesh screen and repeated pipetting, the cells were washed twice with PBS. Total RNA was extracted from the differentiated cells of all groups using TRIzol solution (cat. no. 15596-026; Invitrogen, Carlsbad, CA, USA) according to the manufacturer's instructions. cDNA was synthesized from $1 \mu \mathrm{g}$ total RNA using random primers and PrimeScript reverse transcriptase (part of iScript cDNA synthesis kit; cat. no. 170-8890; Bio-Rad, Hercules, CA, USA). Quantitative PCR reactions for the indicated genes were performed using an iScript cDNA synthesis kit and a fluorescent temperature cycler (C1000 Thermal Cycler; Bio-Rad). The primers sequences are listed in Table I.

Immunofluorescence staining. The retrieved samples were embedded in optimum cutting temperature (OCT) compound (Tissue-Tek; Sakura Finetek, Torrance, CA, USA) and frozen. The $4-\mu \mathrm{m}$ frozen sections were fixed in $4 \%$ paraformaldehyde in PBS for $10 \mathrm{~min}$ at room temperature. In order to detect cytoplasmic proteins, the sections were permeabilized with $0.1 \%$ Triton $\mathrm{X}-100$ for $10 \mathrm{~min}$ at room temperature, $5 \%$ goat serum and $1 \%$ bovine serum albumin which was used for blocking. The sections were incubated with the following primary antibodies overnight at $4^{\circ} \mathrm{C}$ : alpha fetoprotein (AFP; AF5134; Affinity, Cambridge, UK), albumin (ALB; ab8940; Abcam, Cambridge, UK) and cytokeratin 19 (CK19; AF0192; Affinity). Following incubation with the primary antibodies, the cells were washed 

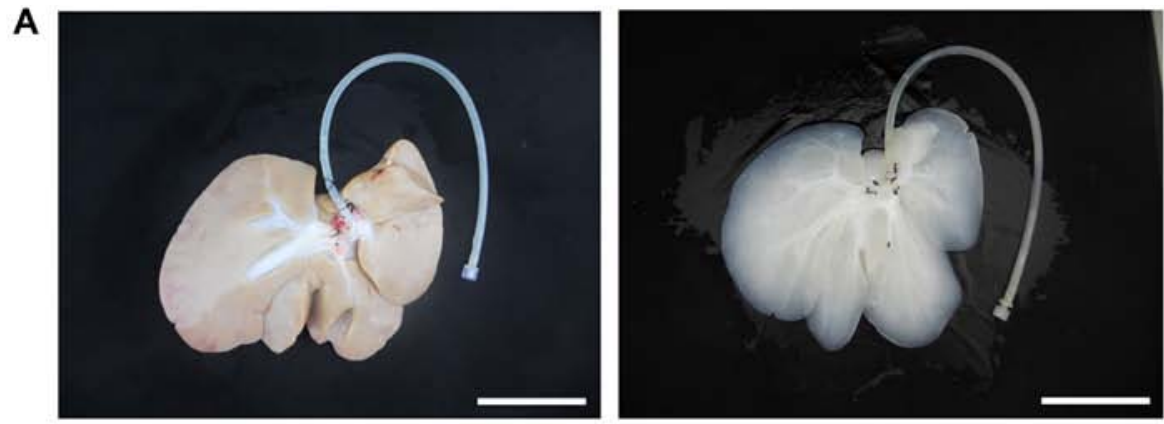

B
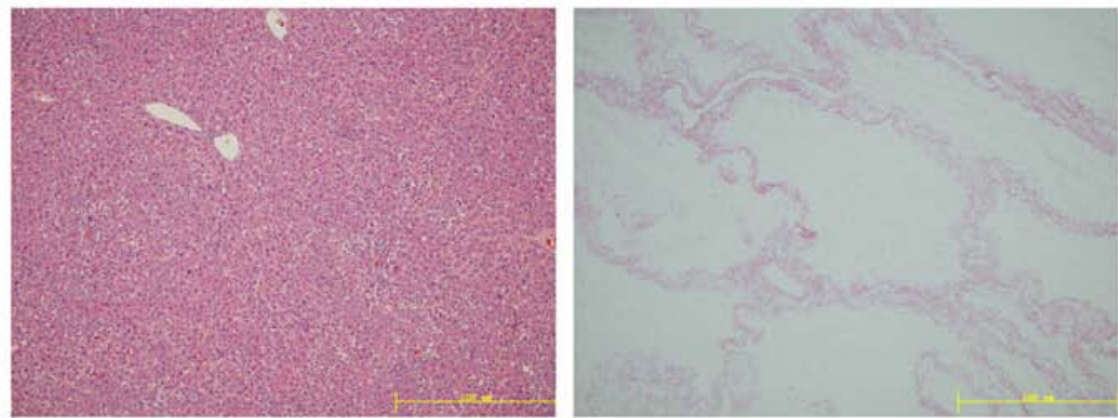

C
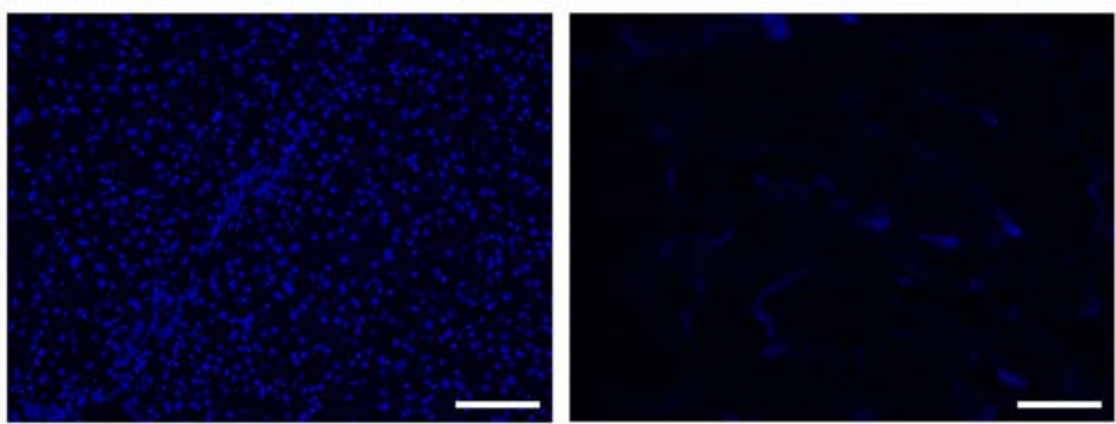

D
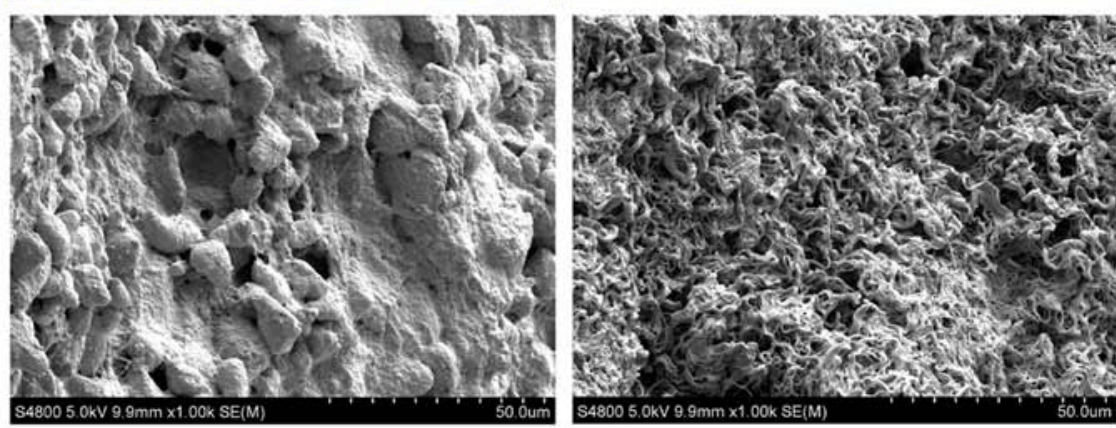

Figure 1. Whole-organ porcine liver decellularization. Representative images of (A) macrograph, (B) H\&E staining, (C) DAPI staining and (D) SEM analysis of the liver ultrastructure. The left panels are images captured prior to decellularization (included as a control) and the right panels are images captured after decellularization. During the liver harvest process, the proximal PV was catheterized and the tubing was retained until the decellularization process completed. Scale bars, (A) $10 \mathrm{~cm}$, (B and C) $100 \mu \mathrm{m}$ and (D) $50 \mu \mathrm{m}$.

with PBS and then incubated with fluorescence-conjugated secondary anti-goat IgG or anti-chicken $\operatorname{IgG}$ (Abcam) for $1 \mathrm{~h}$ at room temperature. After nuclear staining with DAPI, the slides were mounted and analyzed with a fluorescence microscope (Leica DMI 6000; Leica, Mannheim, Germany).

\section{Hepatocyte-specific function assays}

Albumin and urea production. The conditioned media from the differentiated BM-MSCs of all groups were collected on day 21 and stored at $-20^{\circ} \mathrm{C}$ until used for assaying. The albumin level was tested using an ELISA kit (Rat Albumin ELISA Quantitation set, E110-125; Bethyl Laboratories, Inc., Montgomery, TX, USA), according to the manufacturer's instructions. The urea concentration was measured using the QuantiChrom Urea assay kit (DIUR-500; Bioassay Systems LLC, Hayward, CA, USA), and absorbance was measured using a Sunrise microplate reader (MQX 200; BioTek, Winooski, VT, USA). All values were normalized to the number of cells.

Periodic acid-Schiff (PAS) staining. Glycogen storage in the induced BM-MSCs of all groups was determined using a PAS kit (Jiancheng, Nanjing, China) according to the manufacturer's instructions.

Statistical analysis. All data were analyzed using SPSS statistical software (version 17.0). Data are presented as the means \pm SEM. One-way analysis of variance (ANOVA) for multiple compari- 
A

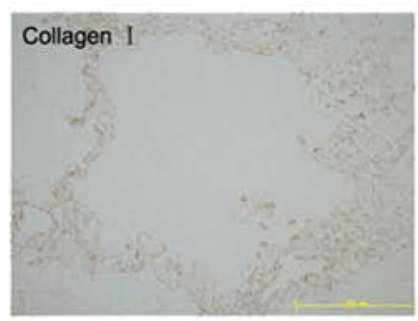

B

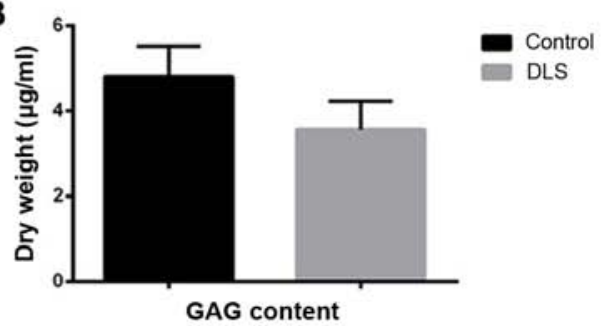

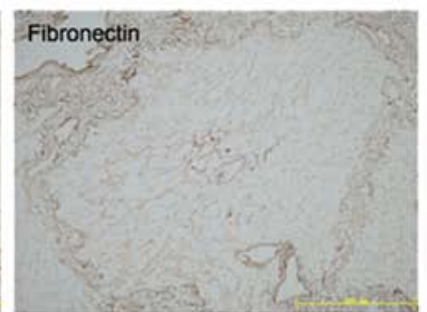

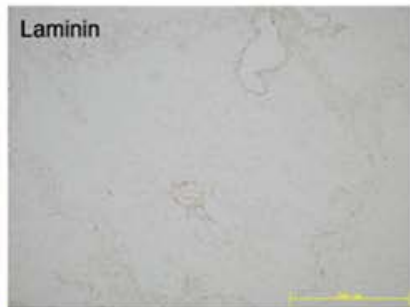

C

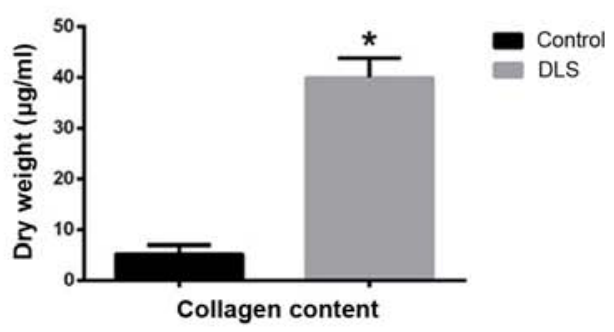

Figure 2. Retention of extracellular matrix (ECM) components by decellularized liver scaffolds (DLSs). (A) Immunohistochemical staining: collagen I, IV, laminin and fibronectin. (B) The sulfated glycosaminoglycan (GAG) content in three group scaffolds. (C) The collagen content DLSs and native liver ( $\mathrm{n}=3$ of each group). "p<0.05 vs. control group.

sons was performed to compare datasets. Dunnett's analysis was performed to compare the two groups' datasets. A p-value $<0.05$ was considered to indicate a statistically significant difference.

\section{Results}

Characterization of decellularized porcine liver. Whole-organ decellularization was achieved through portal perfusion, using sodium dodecyl sulfate (SDS) and Triton X-100. This treatment effectively lyses cell membranes, disrupts intracellular organelles, and removes cellular debris from the tissue. After decellularization, the porcine liver parenchyma became semi-transparent, and the acellular scaffold retained the gross appearance and size of the liver (Fig. 1A). H\&E staining revealed the presence of pink staining, which is typical of collagen, whereas the blue staining typical of cellular nuclear material was not observed (Fig. 1B). The lack of DAPI staining in the biomatrix confirmed the absence of cell nuclei (Fig. 1C). We examined decellularized tissue sections by SEM in order to evaluate whether the ultrastructure of the bio-scaffold was preserved after decellularization (Fig. 1D). Reticular collagen fibers, which provide support for the hepatic tissue, are readily apparent. Immunostaining of the four ECM proteins, namely collagen type I, collagen type IV, fibronectin, and laminin, indicated that the structural components and basement membrane composition of the ECM had been retained (Fig. 2A). There was also a reduction in sulfated GAG content in the decellularized liver tissue (Fig. 2B). The collagen content in the decellularized liver tissue was noted to be significantly higher $(\mathrm{p}<0.05)$ than that found in the fresh liver tissue, which may be explained by the removal of cellular material (Fig. 2C).

$3 D$ spheroid formation. P6-8 BM-MSCs were harvested in order to examine the formation of 3D spheroids (Fig. 3A). As previously described (24), under optimized cell number and growth conditions, BM-MSC aggregates were allowed to form through the forced aggregation method for 2 days (Fig. 3B). In addition, BM-MSC differentiation to the hepatic cell lineage was maintained in monolayer cell culture at a similar cell concentrations. H\&E staining revealed that the cells in spheroids closely adhered to each other and were compacted (Fig. 3C). The Live/Dead staining assay revealed that cells in the 3D spheroids maintained high viability, whereas individual cells not in clusters were no longer viable (Fig. 3D-F). In order to assess cell proliferation within the BM-MSC spheroids, samples were collected for an EdU assay on day 2. EdU staining of the BM-MSC spheroids demonstrated the presence of proliferating cells within the spheroids on day 2. However, EdU-positive cells comprised only a small number of the cells within spheroids, indicating that only a small percentage of the cells $(<5 \%)$ was actively proliferating in 3D culture (Fig. 3G-I). The spheroids were found to have an average diameter of $100 \mu \mathrm{m}$ (Fig. 3J).

Hepatic gene and protein expression of MSC-derived cells. To determine whether the 3D spheroid culture in the DLS promoted hepatocyte maturation of the hepatocyte-like cells, the transcription levels of various genes associated with hepatic development were examined. A comparison of gene expression during the hepatic differentiation of BM-MSCs within the 2D culture, 2D + DLS culture, 3D culture and 3D + DLS culture was performed using RT-qPCR. The transcription levels of various genes associated with hepatic development, namely hepatocyte nuclear factor $1 \beta(H N F 1 \beta)$, hepatocyte nuclear factor 6 (HNF6), AFP, CK19, ALB, tyrosine aminotransferase $(T A T)$, forkhead box A1 (FOXAl), arginase 1 (ARGl), and the members of the cytochrome $\mathrm{P} 450$ subunits cytochrome P450, family 1 , subfamily A, members 1 and 2 (CYPIAl and CYP1A2) were examined. The expression levels of these genes were similar in the 3D and 2D + DLS groups, and levels in both were clearly higher than in traditional $2 \mathrm{D}$ culture. We found that the relative gene expression levels of differentiating BM-MSCs within the 3D + DLS group after 3 weeks of hepatic induction were significantly higher than the other groups $(\mathrm{p}<0.05)$, which indicated that 3D spheroid culture in the DLS provided a preferable external environment for differentiation (Fig. 4). Immunofluorescence analysis was then performed to reveal 

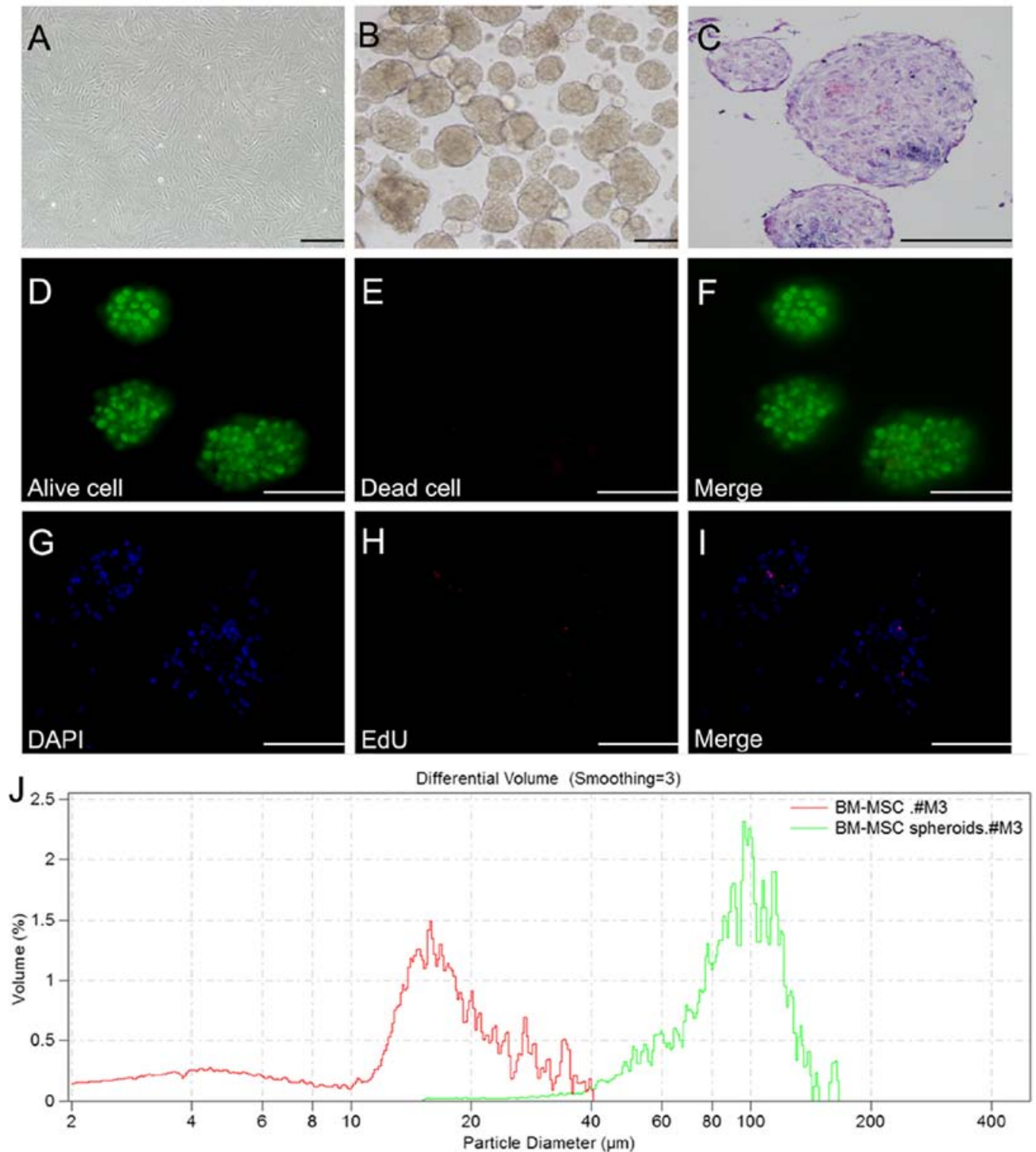

Figure 3. Three-dimensional culture of bone marrow-derived mesenchymal stem cells (BM-MSCs) in the Rocker system. (A) View of BM-MSC two-dimensional (2-D) culture. (B) Spheroids after 48-h Rocker culture. (C) H\&E staining of spheroids. (D-F) Cell viability in spheroids measured by FluoroQuench staining. (G-I) 5-Ethynyl-2'-deoxyuridine (EdU) proliferation assay in spheroids. (J) Diameter analysis of the spheroids measured using a Multisizer 3. Scale bar, $100 \mu \mathrm{m}$.

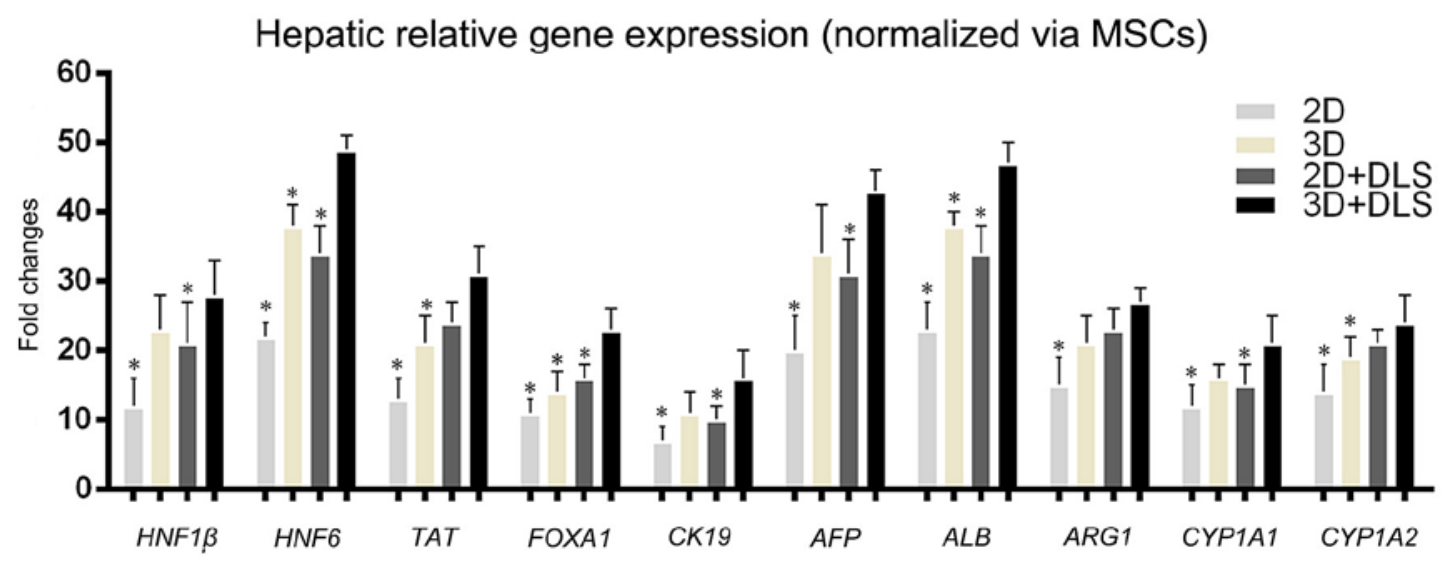

Figure 4. Hepatic-related gene expression analysis measured by RT-qPCR. Comparison of gene transcription levels after 3 weeks of hepatic induction among all the groups. Hepatocyte nuclear factor $1 \beta(H N F 1 \beta)$, hepatocyte nuclear factor $6(H N F 6)$, alpha fetoprotein $(A F P)$, cytokeratin $19(C K 19)$, albumin $(A L B)$, tyrosine aminotransferase (TAT), forkhead box A1 (FOXA1), arginase 1 (ARG1), and the members of the cytochrome P450 subunits, family 1, subfamily A, members 1 and 2 (CYP1A1 and CYP1A2) were examined. Statistically significant differences relative to levels of undifferentiated mesenchymal stem cells (MSCs) using the two-dimensional (2D) approach, which were arbitrarily set to 1.0 , are indicated. " $\mathrm{p}<0.05$ vs. three-dimensional (3D) + decellularized liver scaffold (DLS) group. 

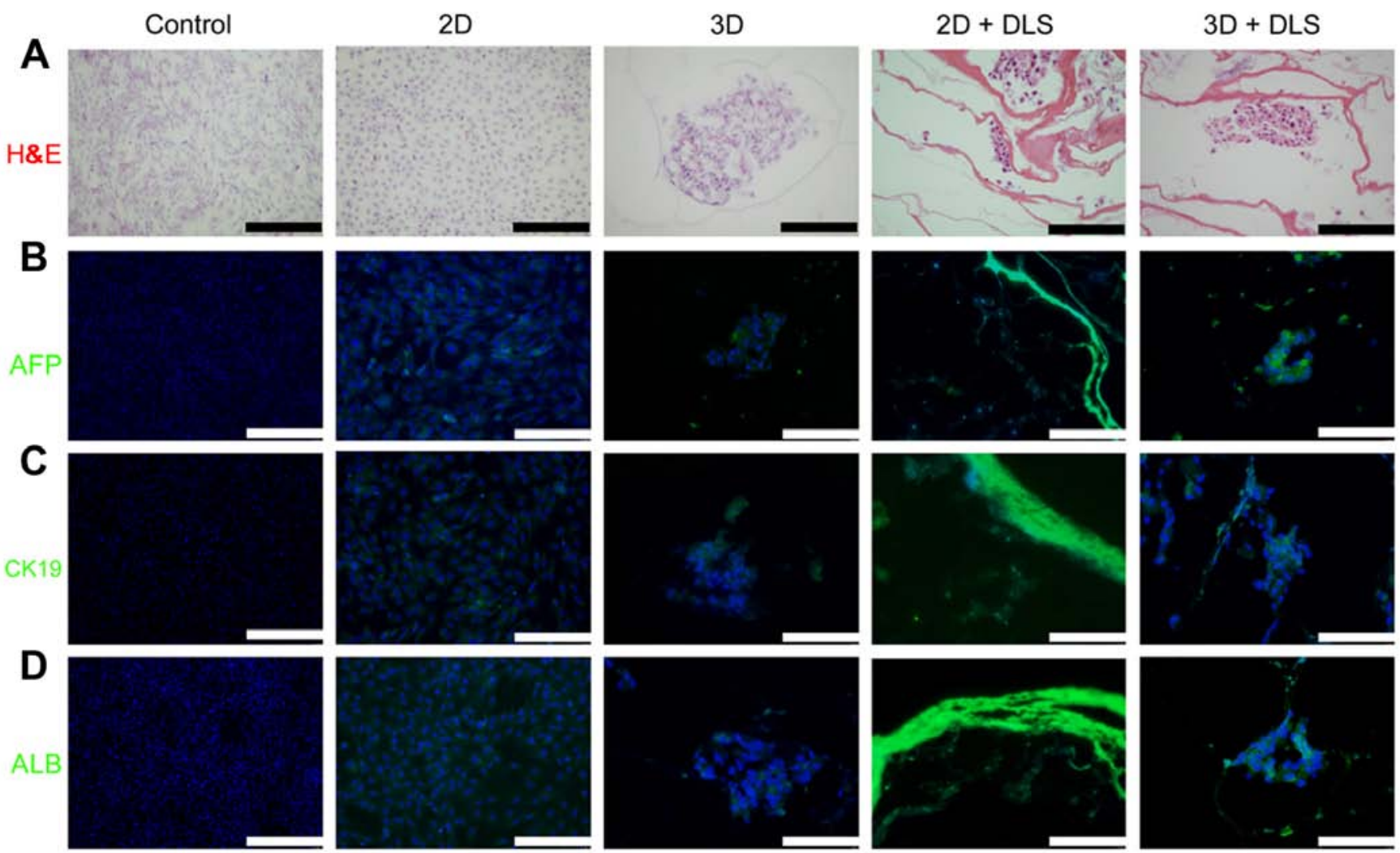

Figure 5. Analysis of hepatic protein markers after 3 weeks of hepatic differentiation. (A) H\&E staining of hepatic differentiation of mesenchymal stem cells (MSCs) in all groups. Immunofluorescence analysis of (B) alpha fetoprotein (AFP), (C) cytokeratin 19 (CK19) and (D) albumin (ALB) expression in all groups. Undifferentiated MSCs were used as controls. Scale bar, $100 \mu \mathrm{m}$. 2D, 2-dimensional; 3D, 3-dimensional; DLS, decellularized liver scaffold.

A

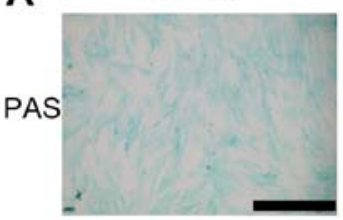

B

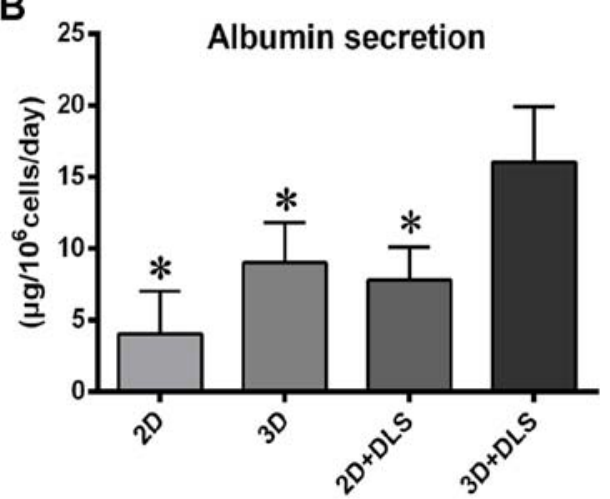

$3 \mathrm{D}$

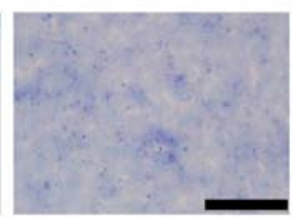

C

C
$2 D+D L S$
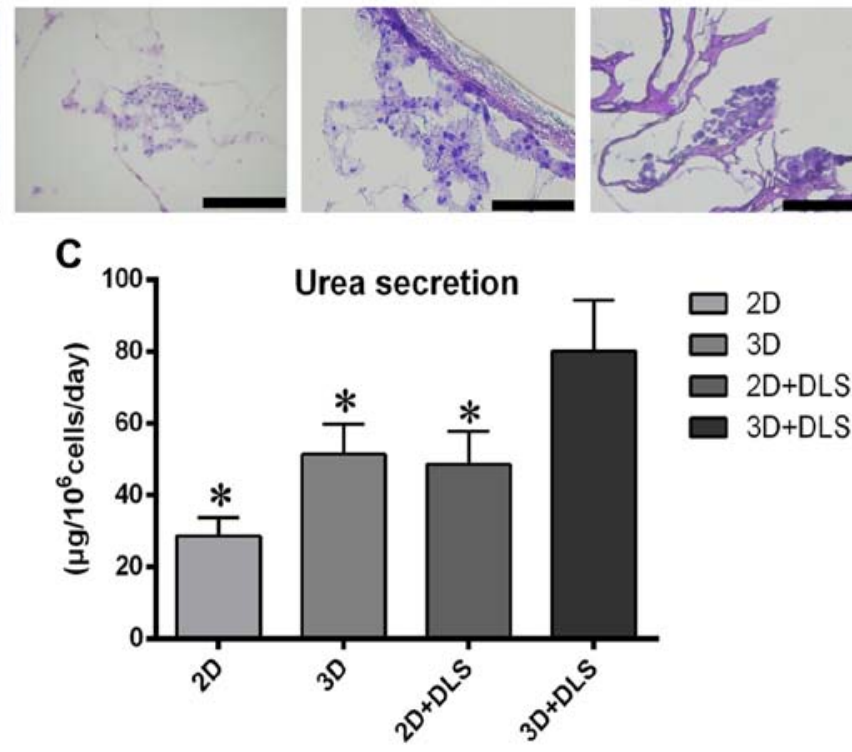

Figure 6. Hepatic function analysis after 3 weeks of hepatic differentiation. (A) Periodic acid-Schiff (PAS) staining indicated the glycogen storage capability in all groups. Undifferentiated mesenchymal stem cells (MSCs) were used as controls. Scale bar, $100 \mu \mathrm{m}$. (B) Albumin secretion and (C) urea production after hepatic differentiation for 3 weeks in all groups. " $\mathrm{p}<0.05$ vs. three-dimensional (3D) + decellularized liver scaffold (DLS) group. 2D, 2-dimensional.

the expression of various hepatic progenitor protein markers, namely AFP, CK19 and ALB (Fig. 5). The high AFP and CK19 expression observed in the cells in the $3 \mathrm{D}+$ DLS group may represent the phenotype of hepatic progenitors, which is consistent with the RT-qPCR results.
Functional analysis of differentiated BM-MSCs. We evaluated a number of hepatic functions in the differentiated BM-MSCs in all groups. PAS staining revealed that after hepatic differentiation, the 3D + DLS group had the highest glycogen storage capability (Fig. 6A). To evaluate metabolic activity, we quanti- 
fied ALB secretion and urea production by the differentiated BM-MSCs in all four groups after 3 weeks of hepatic induction. The cumulative ALB level of the differentiated cells in the $3 \mathrm{D}+\mathrm{DLS}$ group was significantly higher than in the other groups $(\mathrm{p}<0.05)$ (Fig. 6B). The urea concentration in the media produced by the differentiated cells in the $3 \mathrm{D}+$ DLS group was markedly higher than that in the $2 \mathrm{D}$ culture media over the same period of time $(\mathrm{p}<0.05)$ (Fig. 6C). Taken together, these findings clearly demonstrate the superiority of the $3 \mathrm{D}$ spheroid culture on the DLS in terms of supporting the hepatic differentiation of BM-MSCs.

\section{Discussion}

Functional hepatocytes may be exploited for clinical and scientific applications. The generation of stem cell-derived hepatocytes holds considerable promise for future clinical applications. However, there remain some obstacles to obtaining abundant supplies of hepatocyte-like cells. In the present study, a natural 3D scaffold from decellularized liver matrix was used to optimize the differentiation of MSC spheroids to functional hepatocytes. Our data demonstrated that the 3D biomatrix scaffold as well as spheroid culture promotes the induction of lineage-specific differentiation of MSCs into hepatocyte-like cells, which is consistent with the findings of other studies (15-17,20,21). Moreover, we suggest that the combination of cell-cell interactions and ECM plays a positive role in the differentiation of MSCs to hepatocyte-like cells.

3D spheroid cultures are useful for maintaining primary hepatocyte functions, which may be enhanced by effective cellcell interactions. In our experiments, 3D culture spheroids were generated from BM-MSCs using a novel rocking culture system. It is known that the size of the spheroid is important for generating spheroids with high viability, and spheroids up to $100 \mu \mathrm{m}$ in diameter are not oxygen limited (25). To prevent necrosis of the core caused by a deficiency of oxygen and nutrients, the diameter of the spheroid was controlled by the cell seeding density, culture time and the rocking speed. Previous 3D spheroid culture systems often involved a cell-accumulation technique in microwells with certain types of cells $(15,16)$. However, these culture systems experienced difficulties in terms of producing sufficient numbers of MSC spheroids. Our Rocker system is therefore a convenient device that forms abundant spheroids from single MSCs.

Compared to the $2 \mathrm{D}$ culture system, the aggregate culture yielded more hepatocyte-like cells using the same culture volume. Moreover, it promoted higher transcript levels of hepatocyte-specific genes and more mature functions in the final stages of differentiation. This may have been due to cell-cell interactions, which are observed in the native environment of hepatocytes. In addition, the functional polarity of cells in spheroids has been shown to help in enhancing and stabilizing the differentiated functions of hepatocytes (26). Whether similar positive effects of 3D culture on hepatocytes are involved in enhancing the differentiation of stem cells certainly warrants further investigation. Taken together, these findings indicate that the 3D culture provides more suitable conditions for producing hepatocyte-like cells than the monolayer culture.

Whole-organ decellularization is an attractive technique for the preparation of a natural biomatrix scaffold. To date, the potential application of this technique has been demonstrated successfully for a number of organs, including the heart, lung, liver, kidney and bladder (27). A decellularized native liver-derived bioscaffold may provide a suitable environment for differentiation by providing not only a 3D structure but also by maintaining bioactive molecules. Thus, decellularized organs may potentially be used as a tool for stem cell differentiation and maturation to eventually engineer autologous liver grafts for transplantation, as previously described $(20,21)$. Triton X-100 is usually used to solubilize cellular membranes, and SDS is used to clear the remaining nuclear remnants from the matrix. It has been noted previously that the order of detergents used in the decellularization procedure has an effect on retaining the ECM (28). In the present study, we utilized a unique protocol based on a Triton-SDS-Triton perfusion to prepare the decellularized liver, creating a translucent liver matrix within a relatively short period of time in which the porous architecture and partial ECM of the original organ was preserved.

The composition and concentration of ECM proteins are important for cell attachment, growth and differentiation (19). Although it has been reported that the liver biomatrix scaffold exhibited independent inductive potential for the differentiation of MSCs into cells of hepatic lineage, hepatic growth factors and cytokines were adopted in the differentiation protocol of the seeded cells in all groups in order to achieve maximal hepatic induction (20). When compared with the 2D culture system, the extensive analyses of synthetic and metabolic functions demonstrated that the MSCs cultured in the DLS exhibited more abundant and stable functions. The DLS generated in this study is capable of efficiently promoting the hepatic differentiation of MSCs, implying high efficiency in mass transfer. These results suggest that the hepatic maturation of the differentiated BM-MSCs was higher in the DLS than in monolayer cultures.

$3 \mathrm{D}$ cell culture systems are thought to more closely resemble the physiological tissue environment by enabling greater cell-cell and cell-matrix interactions than conventional monolayer culture techniques. 3D spheroid cultures and 3D DLS cultures provide different functional supports for MSC differentiation. To the best of our knowledge, this study is the first to use a combination of these two culture techniques to successfully generate functional hepatocyte-like cells from MSCs. The upregulation of hepatic-enriched transcription factors (HNF1 $\beta, H N F 6$ and FOXA1), hepatic progenitor marker proteins ( $A F P$ and $C K 19)$, liver-associated enzymes (TAT and $A R G 1)$, plasma protein $(A L B)$, and conjugating enzymes ( $C Y P 1 A 1$ and $C Y P 1 A 2)$ was observed in the MSC spheroids cultured on DLSs, and the expression levels were significantly higher than in the other groups. Moreover, the protein expression and hepatic-specific functions confirmed the hepatic differentiation of the MSC spheroids in the DLS culture. These results suggest that this culture combination promotes the hepatic differentiation of murine MSCs into high yields of mature hepatocytes.

In conclusion, in this study we discussed the in vitro production of functional hepatocytes from BM-MSC spheroids on DLSs. Our findings may have future applications in stem cell-based liver regenerative medicine for the treatment of liver injuries and the establishment of a bioartificial liver. 


\section{Acknowledgements}

The present study received funding from the National Natural Scientific Foundations of China (no. 81200315), and the Sichuan Province Science and Technology Support Project (no. 2013SZ0080).

\section{References}

1. Brown RS Jr: Live donors in liver transplantation. Gastroenterology 134: 1802-1813, 2008.

2. Vosough M, Moslem M,Pournasr B and Baharvand H: Cell-based therapeutics for liver disorders. Br Med Bull 100: 157-172, 2011.

3. Nussler A, Konig S, Ott M, Sokal E, Christ B, Thasler W, Brulport M, Gabelein G, Schormann W, Schulze M, et al: Present status and perspectives of cell-based therapies for liver diseases. J Hepatol 45: 144-159, 2006.

4. Zamule SM, Coslo DM, Chen F and Omiecinski CJ: Differentiation of human embryonic stem cells along a hepatic lineage. Chem Biol Interact 190: 62-72, 2011.

5. Shafritz DA, Oertel M, Menthena A, Nierhoff D and Dabeva MD: Liver stem cells and prospects for liver reconstitution by transplanted cells. Hepatology 43 (Suppl 1): S89-S98, 2006.

6. Parekkadan B and Milwid JM: Mesenchymal stem cells as therapeutics. Annu Rev Biomed Eng 12: 87-117, 2010.

7. Schwartz RE, Reyes M, Koodie L, Jiang Y, Blackstad M, Lund T, Lenvik T, Johnson S, Hu WS and Verfaillie CM: Multipotent adult progenitor cells from bone marrow differentiate into functional hepatocyte-like cells. J Clin Invest 109: 1291-1302, 2002.

8. Hong SH, Gang EJ, Jeong JA, Ahn C, Hwang SH, Yang IH, Park HK, Han $\mathrm{H}$ and Kim $\mathrm{H}$ : In vitro differentiation of human umbilical cord blood-derived mesenchymal stem cells into hepatocyte-like cells. Biochem Biophys Res Commun 330 $1153-1161,2005$

9. Lange C, Bassler P, Lioznov MV, Bruns H, Kluth D, Zander AR and Fiegel HC: Hepatocytic gene expression in cultured rat mesenchymal stem cells. Transplant Proc 37: 276-279, 2005.

10. Chivu M, Dima SO, Stancu CI, Dobrea C, Uscatescu V, Necula LG, Bleotu C, Tanase C, Albulescu R, Ardeleanu C and Popescu I: In vitro hepatic differentiation of human bone marrow mesenchymal stem cells under differential exposure to liver-specific factors. Transl Res 154: 122-132, 2009.

11. Lee KD, Kuo TKC, Whang-Peng J, Chung YF, Lin CT, Chou SH, Chen JR, Chen YP and Lee OK: In vitro hepatic differentiation of human mesenchymal stem cells. Hepatology 40: 1275-1284, 2004.

12. Tong JZ, Sarrazin S, Cassio D, Gauthier F and Alvarez F: Application of spheroid culture to human hepatocytes and maintenance of their differentiation. Biol Cell 81: 77-81, 1994.

13. Li Y, Guo G, Li L, Chen F, Bao J, Shi YJ and Bu H: Threedimensional spheroid culture of human umbilical cord mesenchymal stem cells promotes cell yield and stemness maintenance. Cell Tissue Res 360: 297-307, 2015.

14. Frith JE, Thomson B and Genever PG: Dynamic three-dimensional culture methods enhance mesenchymal stem cell properties and increase therapeutic potential. Tissue Eng Part C Methods 16: 735-749, 2010.
15. Subramanian K, Owens DJ, O'Brien TD, Verfaillie CM and $\mathrm{Hu}$ WS: Enhanced differentiation of adult bone marrow-derived stem cells to liver lineage in aggregate culture. Tissue Eng Part A 17: 2331-2341, 2011 .

16. Subramanian K, Owens DJ, Raju R, Firpo M, O'Brien TD, Verfaillie CM and Hu WS: Spheroid culture for enhanced differentiation of human embryonic stem cells to hepatocyte-like cells. Stem Cells Dev 23: 124-131, 2014.

17. Takayama K, Kawabata K, Nagamoto Y, Kishimoto K, Tashiro K, Sakurai F, Tachibana M, Kanda K, Hayakawa T, Furue MK and Mizuguchi H: 3D spheroid culture of hESC/hiPSC-derived hepatocyte-like cells for drug toxicity testing. Biomaterials 34 : 1781-1789, 2013.

18. Ng SL, Narayanan K, Gao S and Wan AC: Lineage restricted progenitors for the repopulation of decellularized heart. Biomaterials 32: 7571-7580, 2011.

19. Wang Y, Cui CB, Yamauchi M, Miguez P, Roach M, Malavarca R, Costello MJ, Cardinale V, Wauthier E, Barbier C, et al: Lineage restriction of human hepatic stem cells to mature fates is made efficient by tissue-specific biomatrix scaffolds. Hepatology 53: 293-305, 2011.

20. Ji R, Zhang N, You N, Li Q, Liu W, Jiang N, Liu J, Zhang H, Wang D, Tao K and Dou K: The differentiation of MSCs into functional hepatocyte-like cells in a liver biomatrix scaffold and their transplantation into liver-fibrotic mice. Biomaterials 33: 8995-9008, 2012.

21. Jiang WC, Cheng YH, Yen MH, Chang Y, Yang VW and Lee OK: Cryo-chemical decellularization of the whole liver for mesenchymal stem cells-based functional hepatic tissue engineering. Biomaterials 35: 3607-3617, 2014.

22. Wu Q, Bao J, Zhou YJ, Wang YJ, Du ZG, Shi YJ, Li L and Bu H: Optimizing perfusion-decellularization methods of porcine livers for clinical-scale whole-organ bioengineering. Biomed Res Int 2015: 785474, 2015.

23. Bao J, Fisher JE, Lillegard JB, Wang W, Amiot B, Yu Y, Dietz AB, Nahmias Y and Nyberg SL: Serum-free medium and mesenchymal stromal cells enhance functionality and stabilize integrity of rat hepatocyte spheroids. Cell Transplant 22: 299-308, 2013

24. Cheng NC, Wang S and Young TH: The influence of spheroid formation of human adipose-derived stem cells on chitosan films on stemness and differentiation capabilities. Biomaterials 33: 1748-1758, 2012

25. Glicklis R, Merchuk JC and Cohen S: Modeling mass transfer in hepatocyte spheroids via cell viability, spheroid size, and hepatocellular functions. Biotechnol Bioeng 86: 672-680, 2004.

26. Haouzi D, Baghdiguian S, Granier G, Travo P, Mangeat P and Hibner U: Three-dimensional polarization sensitizes hepatocytes to Fas/CD95 apoptotic signalling. J Cell Sci 118: 2763-2773, 2005.

27. Crapo PM, Gilbert TW and Badylak SF: An overview of tissue and whole organ decellularization processes. Biomaterials 32: 3233-3243, 2011.

28. Sabetkish S1, Kajbafzadeh AM, Sabetkish N, Khorramirouz R, Akbarzadeh A, Seyedian SL, Pasalar P, Orangian S, Beigi RS, Aryan Z, et al: Whole-organ tissue engineering: decellularization and recellularization of three-dimensional matrix liver scaffolds. J Biomed Mater Res A 103: 1498-1508, 2015. 